\section{Substanz und Supplement}

\author{
Mit Rechten reden, zu Rechten forschen? Eine Einladung zum Widerspruch
}

Robert Feustel

,Substanz und Supplement. Mit Rechten reden, zu Rechten forschen?"

Kommentare von: Regina Ammicht Quinn, Lee Hielscher, Annekatrin Kühn, Katrin Lehn, Nils Kumkar, Daniel Mullis, Andreas Nölke

Replik von: Robert Feustel

Robert Feustel

Die Frage, ob und wenn ja wie mit Rechten zu reden wäre, ist gegenwärtig einigermaßen prominent. Dass der Versuch, rechte Akteure argumentativ umzustimmen oder zu überzeugen, wenig erfolgversprechend ist, hat sich zudem herumgesprochen. Doch was heißt das für die Forschung? Der Beitrag diskutiert kritisch die Möglichkeiten und Grenzen empirischer Sozialforschung an und mit Rechten. Einerseits zeigt sich, dass die Forschung möglicherweise nur Wissensbestände aktualisiert, die schon bekannt sind. Und andererseits könnte es aufgrund der sich rasant verändernden technischen, sozialen und kulturellen Rahmenbedingungen wichtig sein, der Theorie mehr Gehör zu schenken und den Versuch zu starten, die überlieferten theoretischen Kategorien zu überdenken.

Seit rechte Parteien und Bewegungen bei Wahlen zulegen beziehungsweise an Einfluss gewinnen, wird die Frage diskutiert, ob und wenn ja wie mit Rechten geredet werden müsse. Zumeist geht es um sachliche, soziale und moralische Abgrenzungen, um taktische oder strategische Fragen: Ist die Gesprächsverweigerung, besonders in Form moralischer Überlegenheit Wasser auf die Mühlen der Rechten und ihrer Opferinszenierung? Oder verhilft man demagogischer Hasssprache zu mehr Reichweite, wenn ihr eine Bühne oder ein Podium geboten wird? Kurz: Hat das moralische oder sachliche Argument eine Chance gegen demagogische Affektmanager? Und nicht zuletzt geht es um persönliche Grenzen: Sind solche Diskussionen erträglich?

Bislang ist die daran anschließende Frage wenig diskutiert worden, ob und wie kritische Wissenschaftler_innen im Forschungsprozess oder auf Podien mit Rechten reden sollten und welche Erkenntnisse daraus abzuleiten sein könnten. Weil sich Sozialwissenschaft systematisch mit Erklärungen sozialer Prozesse auseinandersetzt und der Zulauf zum rechten oder autoritären Populismus eine relevante soziale Entwicklung darstellt, wird es kaum vermeidbar sein, sich noch intensiver als bisher mit den sozialen Trägergruppen auseinanderzusetzen. Und sofern kritische Wissenschaft immer auch die Perspektiven sozialer Akteur_innen einbezieht, wird sie gezwungen sein, auch mit Angehörigen und Sympathisant_innen des entsprechenden politischen Spektrums zu reden. Dabei müssen erkenntnistheoretische und forschungsethische Probleme diskutiert, methodische Schwierigkeiten geklärt und nicht zuletzt Aspekte der Darstellung und Anwendung der Forschung berücksichtigt werden. Kritische Forscher_innen werden in diesem Fall 
zumeist nicht Gefahr laufen, mit den untersuchten Akteur_innen zu sympathisieren, sondern im Gegenteil ihre politische Haltung in die Situation der Datenerhebung hineintragen. Wie ist unter diesen Bedingungen sachliche Distanz und Unvoreingenommenheit möglich? Wie lässt sich moralische Überheblichkeit umgehen und die „empathy wall“ (Hochschild 2016) einreißen? Und wie bleiben Forscher_innen offen für Überraschungen? Welche Methoden und Feldzugänge sind dafür geeignet? Diesen Fragen widmet sich der Debattenteil dieser s u b $\backslash$ u r b a n-Ausgabe. Die folgenden Seiten liefern einen möglichst pointierten Aufschlag, eine erste Annäherung an das Thema, die zugleich als Herausforderung zur Diskussion, zum Widerspruch gedacht ist.

„Argumentieren Sie ruhig mit Pegidas. Sie werden einen Tritt in den Arsch bekommen. Heißt: Die Möglichkeit vernünftiger

Arbeit besteht nur in der Eigengruppe beziehungsweise auf gesellschaftlichen Feldern, auf denen man selber etwas bewirken

kann. Nicht in der Widerlegung prinzipieller Idiotien. Das ist

Zeitverschwendung." (Theweleit/Seeßlen 2016: 47)

\section{Eigentliche Gründe?}

In der politischen und medialen Debatte ist - bei manchen jedenfalls mittlerweile die Einsicht gereift, dass das unablässige Eingehen auf rechtspopulistische Themen entsprechenden Akteur_innen in die Karten spielt, selbst wenn kritische Töne angestimmt werden: Wer beständig solche Inhalte debattiert, hält sie im Zentrum und betreibt unabsichtlich auf die eine oder andere Weise das Geschäft der neuen Rechten.[1] Mitunter wirkt die Beharrlichkeit, mit der Flucht und Migration, Grenzkontrollen et cetera täglich die Medien dominieren wie ein großes Ablenkungsmanöver, wie eine Scharade, hinter der vor allem soziale Ungleichheiten verborgen bleiben. Daraus resultiert die Annahme, dass es zielführend sein könnte, politisch nicht länger ausführlich über Migration, ihre tatsächlichen und fingierten Probleme und andere typisch rechte Themen wie Sicherheit zu reden und das Spielfeld der neuen Rechten zu verlassen. Dies lässt (etwas voreilig) die Vermutung zu, dass auch nicht unbedingt über Rechte geforscht werden muss, jedenfalls nicht unbedingt zur Frage, wie sie ticken und wie sich ihre Einstellungen beschreiben oder erklären lassen.

Zudem weiß die Forschung seit Jahrzehnten, dass Rassismus und autoritäre Charakteranteile überdauern und kein nur modisches oder aktuelles Problem sind. Sie lassen sich eher psychoanalytisch verstehen. Außerdem ist bekannt, dass sich entsprechende Haltungen nur schwer aufklärerisch bearbeiten oder gar austreiben lassen. Und dennoch ist dieser Tage unablässig der Ruf zu vernehmen, nicht nur über, sondern mit den Rechten zu reden - politisch wie wissenschaftlich. Die Frage ist allerdings, was das für neue Erkenntnisse bringen soll, wenn Einstellungen Produkt komplexer, tiefschürfender und langfristiger Strukturen und individueller Dispositionen sind. Welche neuen Argumentationen oder gar Handlungsoptionen, die über 
die theoretischen Einsichten der vergangenen Jahrzehnte hinausgehen, sollen Gespräche oder Befragungen zutage fördern?

Möglicherweise ist die interessantere Frage jene danach, warum entsprechende Haltungen und Politikvorstellungen besonders gegenwärtig Auftrieb haben. Was führt dazu, dass ein rechter Sog an Kraft gewinnt? Wäre es nicht geboten, vor allem diese Umstände zu erforschen, also die Strukturen auf dem Wohnungs-, Arbeits- oder Bildungsmarkt (beziehungsweise überhaupt die marktförmige Organisation all dieser Dinge), die systematischen Ausgrenzungspraktiken und sozialen Strukturen, die dem rechten Autoritarismus in die Karten spielen? Ob dafür rechte Akteur_innen und Anhänger_innen sinnvolle Gesprächspartner_innen sind, ist allerdings zweifelhaft, weil anzunehmen ist, dass die Lücke zwischen der nützlichen agitatorischen Oberfläche und den ,eigentlichen ' Gründen groß sein dürfte.[2] Wer robuste Ausgrenzung, schroffe Freund-Feind-Schemata und vor allem Abwertungsmechanismen braucht, wird schwerlich über die Gründe solcher Praktiken auskunftsfähig sein. Der Blick hinter die rechte Fassade der Akteur_innen scheint eher psychologisch als soziologisch möglich.

Zugegeben: Solche Einwände sind einigermaßen pauschal, weil die Plausibilität entsprechender Forschungspraktiken von der konkreten Fragestellung und der angenommenen Reichweite der erhofften Ergebnisse abhängt. Zudem droht eine Rhetorik der Eigentlichkeit, also der Annahme, dass hinter rassistischen und autoritären Haltungen und Praktiken immerzu Abgründe lauern, die den Menschen selbst verborgen bleiben. Die Anhänger_innen der Rechten (und teils sicherlich auch ihre Intellektuellen) erscheinen dann als letztlich willenlose Schäfchen, die gewissermaßen vom Kontext und vom Unbewussten navigiert werden und deren Aussagen wenig von tatsächlichen Verhältnissen berichten. Solche Perspektiven sind ohne Zweifel reduktionistisch und mindestens ein wenig überheblich, weil sie zwingend die Behauptung mitführen, die Wissenschaft wisse es besser und könne, wenn auch abstrakt oder theoretisch, hinter die Fassade blicken. Will heißen: Die Forschung muss ihre Proband_innen oder Interviewpartner_innen schon ernst nehmen, auch wenn sie Gefahr läuft, Sequenzen rechter Agitation auswerten und damit reproduzieren zu müssen.

\section{Dominanz des Kontrafaktischen}

Über diese wissenschaftlichen oder erkenntnistheoretischen Komplikationen hinweg sind einige Entwicklungen zu konstatieren, die die Erforschung der neuen Rechten mithilfe von empirischer Sozialforschung erschweren dürften.

Erstens, die politische Kultur der Gegenwart hat massiv mit neuen Medien und Kommunikationskanälen zu kämpfen. Wie groß ist die Differenz zwischen dem, was man etwas grob und vorläufig Lebenswirklichkeit (etwa in den Städten) nennen könnte auf der einen Seite, und durch (soziale) Medien konstruierten ,gefühlten Wirklichkeiten' auf der anderen? Fake News und alternative Fakten sind in aller Munde. Spätestens seit 2015 fahren rechtspopulistische Akteur_innen beispielsweise auf dem Ticket massiver Einwanderung, obwohl der Zuzug nie in den propagierten Größenordnungen stattfand, sich länger schon deutlich abgeschwächt hat, eine restriktive und teils brutale Flüchtlingspolitik in Deutschland und Europa dominiert 
und obwohl die permanent beschworenen Horrorszenarien (,Volkstod', ,Scharia' et cetera) ausgeblieben sind. Anders formuliert: Rechtspopulistische Politiken haben oft einen verzerrenden Realitätsbezug. Eine Auswertung von Donald Trumps Twitterkanal würde wenige sinnvolle Erkenntnisse über die US-amerikanische oder globale Wirklichkeit offenbaren.

Die empirische Forschung dürfte also mit einem wissenssoziologischen Problem konfrontiert sein. Ohne Zweifel waren Diskurs- oder Wissenskonstellationen immer schon kompliziert, und die Forschung hat methodisch und theoretisch viel dafür getan, diese selbst zu untersuchen. Allerdings lässt sich vermuten, dass die viel diskutierten neuen technischen Möglichkeiten die Autonomie unterschiedlicher Wissenszusammenhänge deutlich verstärkt hat. Mit den Schlagwörtern Fake News, Echokammer, Filterblase oder confirmation bias werden diese Dinge gegenwärtig heftig diskutiert. Für die praktische Befragung von Akteur_innen könnte dies schwerwiegende Folgen haben, weil nicht mehr nur schräge oder eigenwillige Vermutungen und derbe Zuspitzungen zirkulieren, sondern weil für diese mittlerweile ein ganzer Apparat von Verweisen und Belegen, eine empirisch wenn man so will scheinbar gesättigte Wissensordnung verfügbar ist, aus der sich ein ganzer, kaum verhandelbarer Wissenszusammenhang entsponnen hat. Das idealtypische Gespräch zwischen Forschenden und Interviewten etwa dürfte oft genug jede gemeinsame Faktenbasis genauso vermissen lassen wie Grundlagen vernünftiger Gesprächsführung. Dies hat wenig mit der viel zu oft und viel zu schnell behaupteten Dummheit rechter Akteur_innen zu tun, sondern viel mehr mit einem Zeitgeist, in dem Plausibilität und Redlichkeit durch Marketing,[3] Framing, alternative Fakten und ähnliche Dinge auffällig an den Rand gedrängt wurden. Ohne Zweifel: Gelogen oder vertuscht wurde schon immer. Wenn aber das Kontrafaktische, also die Praxis der offenen, dreisten Behauptung oder Lüge, bis in Parlamente oder Partei- und Regierungszentralen vorgedrungen ist und für die Akteur_innen folgenlos bleibt,[4] haben wissenschaftliche Gütekriterien wenig Raum.

Bei Pegida keifte vor einiger Zeit eine ältere Dame ihre Sorge in eine Kamera, dass sie Weihnachten bald in einer Moschee feiern müsse - ausgerechnet in Dresden. Sollte die Forschung solche Aussagen tatsächlich als Markierung politischer Einstellungen, tatsächlicher Ängste oder was auch immer ernst nehmen? Wir können davon ausgehen, dass der Frau selbst der Unsinn dieser Aussage nicht völlig verborgen bleibt. „Jeder tiefreligiöse Mensch, jeder Neonazi, alle Pegida-Fuzzis wissen doch, dass es Quatsch ist, den sie erzählen“, verdichtet Klaus Theweleit (Theweleit/Seeßlen 2016: 144). Vielleicht stimmt dies nicht im Moment der Aussage selbst, der von Affekten und Emotionen überladen sein dürfte. Zu einem ruhigeren Zeitpunkt allerdings ist eine gewisse Einsicht durchaus anzunehmen. Der Freiraum des bedenkenlosen Behauptens ist der „unschätzbare Vorteil“ rechter Akteur_innen, „das Kontrafaktische [gibt] all ihren Äußerungen die Basis. Es braucht nicht zu stimmen, was sie erzählen. Das eröffnet die Freiheit, alles zu behaupten, was immer ihnen in den Sinn kommt oder ihnen geboten erscheint - eine unschätzbare Freiheit“ (ebd.). Wie soll man mit solchem Gerede wissenschaftlich umgehen, welche Erkenntnisse sollen die Auswertung solcher Daten liefern?

Zweitens, diese disparaten Wissenszusammenhänge haben möglicherweise weitere Folgen. Im Kontext rechtspopulistischer Diskurse wird ein 
Großteil der Wissenschaften als ideologisch voreingenommen verunglimpft. Nicht selten ist von, links-grün-versifften'Akademiker_innen die Rede, die vom System begünstigt würden und dieses nicht kritisieren wollten oder dürften. Leicht erkennbar wird diese Ordnung (böser Staat und böse Wissenschaft gegen freie deutsche Bürger_innen) an den Debatten zu Umweltschutz und Klimaforschung, an der pauschalen Zurückweisung von Queer- und Genderstudies und anderen reaktionären Attacken gegen die Wissenschaften. Wir haben es mit einer hegemonietheoretisch recht gut beschreibbaren Zweiteilung zu tun: Die Wissenschaft als solche wird einer staatstragenden und damit unfreien Äquivalenzkette (Mouffe/Laclau 2014, Nonhoff 2005) zugeordnet (die Elite), womit alle internen Debatten und Streitigkeiten unter den Tisch fallen.

Die empirische Sozialforschung steht also möglicherweise vor dem Problem, dass die Sprecher_innenposition der Wissenschaftlerin oder des Wissenschaftlers von vornherein als unseriös, tendenziös und feindlich wahrgenommen wird. Selbst Werner Patzelt, Politikprofessor an der TU Dresden, Pegida-Sympathisant und linker beziehungsweise kritischer Positionen wahrlich unverdächtig, hatte beim Versuch, zwischen 2015 und 2016 Pegida zu erforschen, auffällig damit zu kämpfen, dass seine Befragung von vielen Teilnehmer_innen gemieden wurde. Beschimpfungen und Anfeindungen waren an der Tagesordnung. Offenbar war die Reaktivierung eines anCarl Schmitt anschließenden Freund-Feind-Denkens ziemlich erfolgreich, das bekanntlich Kernbestandteil der ,Konservativen Revolution“ ist und einer Naturalisierung oder Essentialisierung des Politischen Vorschub leistet. Wenn das forschende Subjekt von den Beforschten als Feind betrachtet wird, wird eine Befragung nach den Standards der Sozialforschung kompliziert.

Drittens, in neurechten Debatten zählt zudem die Differenzierung von moralischen beziehungsweise ethischen und sachlichen Problemdimensionen nicht oder wird anders konzeptualisiert: Was den einen als Rassismus zu schaffen macht, deuten die anderen als legitime Selbstverteidigung und wehren so den Einwand mit einem vermeintlich rationalen Achselzucken ab. Das funktioniert dann, wenn soziale Konstrukte wie Nation oder Volk als biologisch verknotet und damit als natürliche Einheiten gedacht werden, die jederzeit das natürliche Recht zur Selbstverteidigung haben. Diese schroffe Differenz zwischen einer kritischen Wissenschaft und neurechter ,Sachlogik ' hat nicht nur verschiedene Weltbetrachtungen zur Folge, die sich durchaus erforschen lassen könnten. Für die Möglichkeiten und Grenzen empirischer Forschung sind die unterschiedlichen Kategorien, in denen die gleichen Themen verhandelt werden, dennoch von herausragender Bedeutung. Wenn Migration auf der einen Seite ein politisches, soziales oder ethisches Thema ist, und auf der anderen deren Verhinderung eines von vermeintlich natürlichem Artenschutz, von sozialdarwinistischer Selbstverteidigung, dann wird Sinnverstehen genauso schwer wie das Generieren von Datensätzen, aus denen sinnvolle Erkenntnisse über diese kategorialen Differenzen hinaus gewonnen werden können. Zugespitzt haben wir es womöglich mit einem recht grundsätzlichen epistemologischen Bias zu tun, der die empirische Sozialwissenschaft vor schwerwiegende Herausforderungen stellt. Entweder Forschende deuten die Daten auf diese Kategorien hin und 
finden Dinge heraus, die wir im Wesentlichen bereits wissen: Neurechte naturalisieren Kultur und Nation, haben kein Verständnis für Historizität bzw. Hybridität und wollen einen „traditionell geschlossenen, organisch strukturierten und homogenen gesellschaftlichen Raum, der keine Leere zulässt“ (Žižek 2001: 259). Oder man folgt den inhärenten Wegen des neurechten Agitierens und reproduziert es damit. Eine dritte Option wäre sicherlich, Menschen mit entsprechenden Einstellungsmustern etwa nach sozialen oder ökonomischen Kontexten und Bedingungen zu befragen, die unter der Oberfläche ihrer Einstellungen liegen und Gründe für diese liefern könnten. Das mag funktionieren, läuft aber Gefahr, sich ganz und gar der beschriebenen Eigentlichkeitserzählung anzudienen oder bestenfalls einer „Hermeneutik des Verdachts‘ (Paul Ricoeur) nachzujagen. Schließlich wissen wir nie so ganz genau, wie das Verhältnis von sozialen Tatsachen oder Bedingungen und politischen Einstellungen beschaffen ist.

\section{Chaos mit Methode}

Ich möchte an dieser Stelle, im Sinne eines bewusst überspitzten Debattenaufschlags und als Herausforderung zur Gegenrede, die These in die Runde geben, dass das wissenschaftliche Reden mit Rechten entweder nur zu wenigen und kaum neuen Erkenntnissen führt oder methodisch bzw. theoretisch hochgradig kompliziert und letztlich nicht umsetzbar ist. Allzu tiefsinnige Einsichten dürften beim Versuch methodisch sauberer Systematisierungen jedenfalls nicht ans Licht kommen. Was John Law (2010: 148) in Making a Mess with Method ganz allgemein für das Verhältnis von Sozialwissenschaft und Wirklichkeit formuliert, gilt gegenwärtig in Bezug auf die neue Rechte und die eben grob skizzierten Veränderungen vielleicht noch dringlicher: Die Welt „ist größtenteils chaotisch“,[5] und die „gegenwärtigen sozialwissenschaftlichen Methoden [scheitern] an der Beschreibung dieser chaotischen Normalität hoffnungslos (ebd.)." Dieser Tage ist kaum zu unterscheiden, was Substanz und was Supplement, was real, was fake, was Einstellung, affektive Laune, Etikett oder Show ist. Die überlieferten (methodischen) Kategorien wollen, jedenfalls in Teilen, nicht mehr viel bedeuten, was der empirischen Sozialforschung zusetzt. Das Konzept einer zu erforschenden Realität ist zersplittert. Das heißt freilich nicht, dass Methoden unsinnig wären. Jedoch könnte es zielführend sein, die Bedingungen, Register und Kategorien die ihr zugrunde liegen kritisch zu analysieren.

Law (2010: 156) skizziert in diesem Sinne einen „poststrukturalistischen Umweg“ und betont, dass auch die Sozialforschung nicht einfach abbildet, sondern einen performativen Effekt hat, dass sie in dem Moment, in dem sie das eine Erklärungsmuster präsentiert, automatisch gewissermaßen ein anderes „der Sichtbarkeit entzieht“. Wir haben es mit der alten Logik einer „Metaphysik der Präsenz“ zu tun (ebd.: 157), die besonders in der empirischen Forschung noch immer ihr Unwesen treibt. „Realität determiniert Repräsentation“, paraphrasiert Law die üblichen oft impliziten Standards empirischer Forschung und fährt fort:

„Der allgemein gebräuchliche Realismus der Natur- und Sozialwissenschaft geht also davon aus, dass seine Repräsentation durch spezielle 
Berichtsverfahren in dieser oder jener Hinsicht besondere Garantien und Rechte besitzt. Gute Methoden erschaffen einen verlässlichen, repräsentativen Kanal zwischen Realität und Darstellung. Er ist eine Einbahnstraße. Die Welt ist geschaffen, um für sich selbst zu sprechen - Ende der Geschichte.“(Law 2010: 157)

Tatsächlich allerdings ist die Welt nicht nur chaotisch. Der ganze methodische Zauber gleicht eher einem „Taschenspielertrick, denn Realitäten werden gleichzeitig mit Repräsentationen der Realität erschaffen“ (ebd.). Das diskursive Chaos der Gegenwart bestätigt Laws Annahme gewissermaßen, und besonders Einstellungsforschung und Meinungsumfragen haben mitunter mehr performative als beschreibende Kräfte.

Und vielleicht ist die Frage falsch gestellt, ob rechte Einstellungen vorrangig sozioökonomisch bedingt oder doch inhärent mehr sind als nur ein Ventil oder eine Oberfläche. Wenn etwa Sicherheitsdiskurse ganz offen auf der Basis von gefühlter Unsicherheit geführt werden und alle beteiligten Akteur_innen um die Differenz zur Welt da draußen wissen, kann die Sozialforschung nicht mehr erwarten, diesem Treiben mit realistischen Grundlagen ihrer Methodik habhaft zu werden. Überall sprießt das Kontrafaktische, das Gefühlte dominiert, beständig im Wissen darum, dass es empirisch schlicht nicht stimmt (etwa Kriminalität, Sicherheit et cetera), politisch aber funktioniert.

Möglicherweise ist es an der Zeit, der guten alten Theorie, der Begriffsarbeit, den abduktiven Schlüssen (also den Geistesblitzen und schrägen Einfällen) mehr Raum oder Gehör zu verschaffen statt beim Versuch zu verkrampfen, jedes Argument empirisch sättigen und absichern zu wollen. Vielleicht braucht es einen neuen „langen Sommer der Theorie“ (Felsch 2015). Spätestens bei der neuen Rechten jedenfalls scheint die Empirie beziehungsweise die methodisch seriöse Wissenschaft an ihre Grenzen zu stoßen. Wer sich beständig im Kontrafaktischen bewegt, wird kaum von einer faktenbasierten Wissenschaft gewinnbringend zu analysieren sein.

Themenfelder für Theoriearbeit gäbe es einige. Etwa könnte die Frage spannend sein, ob Realität als wissenschaftliche Kategorie überhaupt noch tragfähig ist. Vielleicht taugt das vor längerer Zeit von Jean Baudrillard in die Debatte geworfene Konzept einer Hyperrealität zur Beschreibung der Gegenwart eher, also einer schwer zu entwirrenden Verflechtung von medialen oder digitalen und wirklichen Zeichen und Dingen, die sich auch als „Fiktionalisierung der Wirklichkeit“ (Esposito 2007) umschreiben ließe. Vielleicht wäre es sinnvoll, die tatsächlichen Gründe für die allenthalben zu beobachtende Indifferenz und Beliebigkeit zu ergründen, für die ziemlich oft und völlig zu Unrecht, die Postmoderne، verantwortlich gemacht wird (vgl. Sarasin 2016, Sasse/Zanetti 2017). Und vielleicht ist auch die immerzu als selbstverständlich und real verhandelte Kategorie politische Einstellung vor dem Hintergrund einer Allgegenwart des Kontrafaktischen zweifelhaft.

Das heißt immer noch nicht, dass Methoden sinnlos oder falsch wären. Doch vielleicht ist es ratsam - um noch einmal Law zu zitieren - den veralteten Standpunkt zu verlassen, dass „Realität definitiv und singulär ist, dieser immer wieder und an verschiedensten Orten reproduzierte Standpunkt, auch in Debatten darüber, was als gute sozialwissenschaftliche Methode gilt.“ Vielleicht braucht es „,neue Regeln in der Forschung“ und vielleicht „müssen 
wir begreifen, dass unsere Methoden immer mehr oder weniger widerspenstige Assemblagen sind“ (Law 2010: 165). Dies zeigt sich möglicherweise besonders, wenn die neue Rechte und ihre Fans Forschungsgegenstand werden. Wenn Patzelt etwa Pegida empirisch valide und methodisch abgesichert untersuchen will und die Teilnehmenden als ,besorgte Gutwillige', ,empörte Gutwillige', ,xenophobe Patrioten', ,islamophobe Zuwanderungsgegner oder ,kulturkonservative Xenophobe‘ einteilt, gelegentlich unterstützt von ,Rechtsextremen', dann ist das Chaos, das Methoden anrichten können, einigermaßen augenfällig. Schon richtig, Patzelt ist nicht unbedingt der Standard der Forschung, aber die Dimension des Unsinns lässt zumindest die Vermutung zu, dass Grundsätzliches zur Diskussion stehen könnte. Dichte Beschreibungen und theoretische Deutungen verraten vielleicht mehr als ein stabiles, empirisches Fundament. „Es ist keine Schande zu bekennen, dass auch uns Sozialwissenschaftlern die Sprache versagt, angesichts der Wirklichkeit, die uns überrollt", schrieb Ulrich Beck (2014) in seiner Laudatio auf Zygmunt Bauman.

„Die Sprache der soziologischen Theorien (aber auch der empirischen Forschung) erlaubt uns, uns dem Immergleichen des sozialen Wandels oder der Ausnahme der Krise zuzuwenden, aber sie erlaubt uns nicht, die gesellschaftshistorische Verwandlung der Welt am Beginn des 21. Jahrhunderts auch nur zu beschreiben, geschweige denn sie zu verstehen.“ (Beck 2014)

Mit Rechten im Sinne der Forschung zu reden, wird uns dabei jedenfalls nur bedingt helfen.

Die Publikation dieses Beitrags wurde durch Mittel des Thüringer OpenAccess-Fonds gefördert.

\section{Endnoten}

[1] Wir sprechen von der neuen Rechten im Sinne der gesellschaftlichen Bewegung der letzten Jahre; ,Neue Rechte' hingegen ist ein stehender Begriff für ein rechtes Akteursspektrum, das sich seit Ende der 1970er Jahre beobachten lässt und durch das Konzept des Ethnopluralismus hervortritt. Der Begriffsgebrauch soll zugleich anzeigen, dass eine klare Trennung zwischen alter Neuer Rechter und neuer Rechter nicht immer möglich ist.

[2] Selbstredend hängt die Sinnhaftigkeit solcher Gespräche von der Forschungsfrage ab. Hier geht es eher um grundsätzliche Belange oder Tendenzen, nicht um die Plausibilität einzelner Forschungswege.

[3] Hochinteressant ist etwa das in den USA heftig diskutierte astroturfing, also die Praxis von großen Unternehmen und Interessenverbänden, verdeckt Initiativen zu starten, die wie Graswurzelbewegungen aussehen.

[4] Olaf Scholz konnte als Oberbürgermeister von Hamburg im Sommer 2017 einfach behaupten, dass es keine Polizeigewalt beim G-20-Gipfel gegeben habe, genauso wie Michael Kretschmer im September 2018 den Mob von Chemnitz in seiner Regierungserklärung schlicht leugnet. In beiden Fällen ist die Beweislage sowohl für Polizeigewalt als auch für das Auftreten eines rechten Mobs erdrückend.

[5] Die Zitate stammen aus der deutschen Übersetzung. 


\section{Autor_innen}

Robert Feustel ist Politikwissenschaftler und beschäftigt sich mit politischer Theorie sowie Wissens- und Stadtsoziologie.

robert.feustel@uni-jena.de

\section{Literatur}

Beck, Ulrich (2014): Sinn und Wahnsinn der Moderne, in: tageszeitung (taz), 14.10.2014. Esposito, Elena (2007): Die Fiktion der wahrscheinlichen Realität. Frankfurt am Main: Suhrkamp.

Felsch, Philipp (2015): Der lange Sommer der Theorie: Geschichte einer Revolte; 1960-1990. München: Beck.

Hochschild, Arlie (2016): Strangers in their own Land: Anger and Mourning on the American Right. New York: The New Press.

Law, John (2010): Methodisch(e) Welten durcheinanderbringen. In: Robert Feustel / Maximilian Schochow (Hg.), Zwischen Sprachspiel und Methode. Perspektiven der Diskursanalyse. Bielefeld: transcript, 147-168.

Mouffe, Chantall / Laclau, Ernesto (2014): Hegemony and Socialist Strategy. London: Verso.

Nonhoff, Martin (2005): Politischer Diskurs und Hegemonie: das Projekt „Soziale Marktwirtschaft“. Bielefeld: transcript.

Sarasin, Philipp (2016): \#Fakten. Was wir in der Postmoderne über sie wissen können. https://geschichtedergegenwart.ch/fakten-was-wir-in-der-postmoderne-ueber-siewissen-koennen/ (letzter Zugriff am 27.9.2018).

Sasse, Syliva / Zanetti, Sandro (2017): \#Postmoderne als Pappkamerad. https://geschichtedergegenwart.ch/postmoderne-als-pappkamerad/ (letzter Zugriff am 27.9.2018).

Theweleit, Klaus / Seeßlen, Georg (2016): Ein Volk, ein Reich, kein Syrer. Ein Gespräch mit Georg Seeßlen und Klaus Theweleit, Moderation Marit Hofmann. In: Robert Feustel / Nancy Grochol / Tobias Prüwer / Franziska Reif (Hg.), Wörterbuch des besorgten Bürgers. Mainz: Ventil, 143-149.

Žižek, Slavoj (2001): Die Tücke des Subjekts. Frankfurt am Main: Suhrkamp. 
